\title{
Benzoxazole derivatives suppress lipopolysaccharide-induced mast cell activation
}

\author{
KYUNG-AH CHO $^{1}$, MINHWA PARK ${ }^{1}$, YU-HEE KIM ${ }^{1}$, HEA-YOUNG PARK CHOO ${ }^{2}$ and KYUNG HO LEE ${ }^{3}$ \\ ${ }^{1}$ Department of Microbiology, School of Medicine, Ewha Womans University, Seoul 07985; ${ }^{2}$ College of Pharmacy and \\ Graduate School of Pharmaceutical Sciences, Ewha Womans University, Seoul 03760; ${ }^{3}$ Department of Dermatology, \\ Bucheon St. Mary's Hospital, College of Medicine, The Catholic University of Korea, Bucheon-si 14647, Republic of Korea
}

Received October 18, 2017; Accepted March 6, 2018

DOI: $10.3892 / \mathrm{mmr} .2018 .8719$

\begin{abstract}
Mast cells are central regulators of allergic inflammation that function by releasing various proallergic inflammatory mediators, including histamine, eicosanoids and proinflammatory cytokines. Occasionally, bacterial infections may initiate or worsen allergic inflammation. A number of studies have indicated that activation of lipoxygenase in mast cells positive regulates allergic inflammatory responses by generating leukotrienes and proinflammatory cytokines. In the present study, the effects of benzoxazole derivatives on the lipopolysaccharide (LPS)-induced expression of proinflammatory cytokines, production of histamine and surface expression of co-stimulatory molecules on bone marrow-derived mast cells (BMMCs) were studied. The benzoxazole derivatives significantly reduced the expression of interleukin (IL)-1 $\beta$, IL-6, IL-13, tumor necrosis factor- $\alpha$, perilipin (PLIN) 2, and PLIN3 in BMMCs treated with LPS. Furthermore, histamine production was suppressed in BMMCs treated with LPS, or treated with phorbol-12-myristate-13-acetate/ionomycin. Benzoxazole derivatives marginally affected the surface expression of cluster of differentiation (CD)80 and CD86 on BMMCs in the presence of LPS, although LPS alone did not increase the expression of those proteins. Therefore, benzoxazole derivatives inhibited the secretion of proinflammatory cytokines in mast cells and may be potential candidate anti-allergic agents to suppress mast cell activation.
\end{abstract}

\section{Introduction}

Mast cells (MCs) are secretory cells that are strategically located at host-environment interfaces, including the skin,

Correspondence to: Dr Kyung Ho Lee, Department of Dermatology, Bucheon St. Mary's Hospital, College of Medicine, The Catholic University of Korea, 327 Sosa-ro, Wonmi-gu, Bucheon-si 14647, Gyeonggi-do, Republic of Korea

E-mail: beauty4u@catholic.ac.kr

Key words: mast cells, benzoxazole derivatives, 5-lipoxygenase inhibitors, histamine, inflammation lungs and mucosal surfaces. MCs release granules following activation and are part of the innate immune system, being involved in the first line of defense against pathogens, including bacteria and parasites (1). Activated MCs produce a broad spectrum of inflammatory cytokines, chemokines, lipid compounds and vasoactive amines, all of which are involved in immune responses (2).

Lipopolysaccharide (LPS) released from Gram negative bacteria is of great significance to mast cells, for the innate immune response and, additionally, as it is able to aggravate an existing inflammatory state in allergic airway inflammation via T helper cell 2 (Th2) cytokine production (3). Toll-like receptor (TLR) 4 is a receptor for LPS that serves an important role in inflammation and is a risk factor for MC-mediated asthma. MCs directly respond to LPS by producing inflammatory cytokines including interleukin-1 $\beta$ (IL-1 $\beta$ ), IL-6, IL-13 and tumor necrosis factor- $\alpha$ (TNF- $\alpha$ ) (4). These mediators exert acute inflammatory effects through endothelial cells and leukocytes. Activation of TLR4 by LPS on MCs leads to the generation of Th2 cytokines (e.g. IL-4, IL-5 and IL-13), which enhances the allergic inflammation that may occur in asthma $(5,6)$. In addition to allergic airway inflammation, MCs trigger inflammatory responses in the skin when exposed to LPS. For example, LPS-stimulated MCs serve an important role in edema formation by releasing factors that increase vascular permeability, including histamine and leukotrienes (LTs) (7).

Lipoxygenases (LOXs) are a family of enzymes that catalyze the incorporation of oxygen into unsaturated fatty acids (8). LOXs are expressed in immune and epithelial cells and they are involved in a variety of conditions, including inflammatory skin disease and allergic asthma, wherein they generate LTs and proinflammatory mediators $(9,10)$. MCs were suggested to be one of the principal cell types responsible for the action of 5-/12-LOX during the progression of asthmatic inflammation due to their production of LTs and IL-13 $(11,12)$. Considering that LTs and IL-13 are potent proinflammatory mediators, LOX may serve a role in allergic inflammation mediated by MCs.

Previously, benzoxazoles have been reported to act as 5-lipoxygenase or IL-6 inhibitors (13-16). To investigate the effects of benzoxazole derivatives on the LPS-induced inflammatory response in MCs, the expression of proinflammatory 
cytokines, the levels of histamine secreted from MCs under LPS stimulation, and the cell-surface localization of co-stimulatory molecule in the presence or absence of benzoxazole derivatives was assessed.

\section{Materials and methods}

Synthesis of benzoxazole derivatives. A total of four derivatives with the benzoxazole moiety were synthesized starting from the commercially available 2-amino-4-nitro (or 4-methyl) phenol (Sigma-Aldrich; Merck KGaA, Darmstadt, Germany) and substituted-phenyl isothiocyanate (Sigma-Aldrich; Merck KGaA; Fig. 1). The thiourea 2 was cyclized to benzoxazole anti-itch agent provided by Park [(ICP)-1, 2, 5, and 13, Laboratory of Dr Park, Ewha Womans University, Seoul, Korea] by oxidation with 5 eq $\mathrm{KO}_{2}$ (Alfa Aesar, Haverhill, MA, USA). This procedure to prepare the benzoxazoles had an $80-90 \%$ yield (13-16). For the preparation of ICP-1 and 5, the nitro group was reduced to amino group with catalytic hydrogenation using 5\% Pd/C (Sigma Aldrich; Merck KGaA) as catalyst.

Generation of bone marrow-derived MCs (BMMCs) and in vitro stimulation. For bone marrow cell isolation, 5 female C57BL/6 mice were purchased at 10 weeks of age (weight, 20-22 g) from OrientBio, Inc., (Emsung, Korea). All animals were maintained at $21-23^{\circ} \mathrm{C}$ with $51-54 \%$ humidity under pathogen-free conditions on a 12-h light/dark cycle with free access to food and water. All procedures were approved by the Ewha Womans University College of Medicine Animal Care and Use Committee (Seoul, Korea; ESM 15-0309). Bone marrow cells were isolated from the femurs of female C57BL/6 mice and grown in Iscove's Modified Dulbecco's Medium (IMEM; Welgene, Daegu, Korea) supplemented with $10 \%$ fetal bovine serum (FBS; Welgene), $50 \mu \mathrm{M} \beta$-mercaptoethanol, $2 \mathrm{mM}$ L-glutamine, $100 \mu \mathrm{g} / \mathrm{ml}$ streptomycin and $100 \mathrm{U} / \mathrm{ml}$ penicillin, and $10 \mathrm{ng} / \mathrm{ml} \mathrm{IL-13}$ (PeproTech, Inc., Rocky Hill, NJ, USA). Non-adherent cells were transferred to fresh medium every 2-3 days to remove adherent macrophages and fibroblasts. After 5 weeks, the cells were identified as MCs using flow cytometric analyses of high affinity immunoglobulin $\varepsilon$ receptor subunit $\gamma$ (FceRI) and cluster of differentiation (CD)117 (c-kit) expression and toluidine blue staining. For toluidine blue staining cytospin slides were stained for $3 \mathrm{~min}$ at room temperature. The image was captured by light microscope with magnification, x1,000 (Olympus BX50, Olympus Corporation; Tokyo, Japan). MCs at 5-6 weeks following initiation of culture were used for the experiments. For treatment with LPS, BMMCs were seeded at a density of $6 \times 10^{5}$ cells/well in 96-well plates and stimulated with LPS (Sigma-Aldrich; Merck KGaA) at a concentration of $1 \mu \mathrm{g} / \mathrm{ml}$. A total of four types of benzoxazole derivatives (ICP-1, 2, 5 and 13) were separately added to the BMMCs at a concentration of $10 \mu \mathrm{M}$ each to observe the effects on LPS-induced BMMCs.

Flow cytometry. The surface expression of FceRI and CD117 on BMMCs following 5 weeks of culture was detected by staining with Alexa 488-conjugated anti-FceRI antibody (Clone MAR-1; cat. no. 134329; BioLegend, Inc., San Diego,
CA, USA) and PerCP-conjugated anti-CD117 antibody (Clone 2B8; cat. no. 105821; BioLegend, Inc.) at a concentration of $5 \mathrm{mg} / \mathrm{ml}$ on ice for $30 \mathrm{~min}$ followed by washing using PBS containing $0.5 \% \mathrm{FBS}$ and $0.1 \%$ sodium azide. The surface expression of CD80 and CD86 were additionally analyzed using fluorescein isothiocyanate-conjugated anti-CD80 antibody (Clone 16-10A1; cat. no. 553768; BD Biosciences, San Jose, CA, USA) and phycoerythrin-conjugated anti-CD86 antibody (Clone GL1; cat. no. 553692; BD Biosciences) at a concentration of $5 \mathrm{mg} / \mathrm{ml}$ on ice for $30 \mathrm{~min}$. The level of nonspecific staining was performed using the corresponding isotype antibody as described above; Armenian Hamster IgG (cat. no. 400923; Biolegend, Inc.) for anti-FceRI antibody, Rat IgG2b, $\kappa$ for anti-CD117 antibody (cat. no. 400629; Biolegend, Inc.). Each sample was quantified on a NovoCyte ${ }^{\mathrm{TM}}$ Flow Cytometer (ACEA Biosciences, San Diego, CA, USA) and the data were analyzed using FlowJo software (version 8.7, FlowJo LLC, Ashland, OR, USA).

Reverse transcription-quantitative polymerase chain reaction $(R T-q P C R)$. Total RNA was extracted from BMMCs with TRIzol ${ }^{\circledR}$ reagent (Invitrogen; Thermo Fisher Scientific, Inc., Waltham, MA, USA). Complementary DNA was synthesized by incubation at $30^{\circ} \mathrm{C}$ for $10 \mathrm{~min}$, at $42^{\circ} \mathrm{C}$ for $20 \mathrm{~min}$ and at $99^{\circ} \mathrm{C}$ for 5 min using the First Strand cDNA Synthesis kit (Toyobo Life Science, Osaka, Japan). Amplification was performed in duplicate by 40 cycles of $15 \mathrm{sec}$ denaturation step at $95^{\circ} \mathrm{C}$ and a 1 min amplification and signal acquisition step at $60^{\circ} \mathrm{C}$ using StepOnePlus Real-Time PCR System (Applied Biosystems; Thermo Fisher Scientific, Inc., Waltham, MA, USA) using SYBR $^{\circledR}$ green (Kapa Biosystems, Inc., Wilmington, MA, USA).

All gene expression values were normalized to the expression of the GAPDH gene using the following primers: 5'-GACCTTGTGTCCTCCGCTTAT-3' forward, and 5'-CAA CCGCAATTTGTGGCTC-3' reverse for mouse perilipin 2 (Plin2; 157 bp); 5'-ATGTCTAGCAATGGTACAGATGC-3' forward, and 5'-CGTGGA ACTGATAAGAGGCAGG-3' reverse for mouse Plin3 (114 bp); 5'-GCAACTGTTCCTGAA CTCAACT-3' forward, and 5'-ATCTTTTGGGGTCCGTCA ACT-3' reverse for mouse $I l l b$ (89 bp); 5'-CTGCAAGAG ACTTCCATCCAG-3' forward, and 5'-AGTGGTATAGAC AGGTCTGTTGG-3' reverse for mouse Il6 (131 bp); 5'-CCT GGCTCTTGCTTGCCTT-3' forward, and 5'-GGTCTTGTG TGATGTTGCTCA-3' reverse for mouse $I 13$ (116 bp); 5'-CCT GTAGCCCACGTCGTAG-3' forward, and 5'-GGGAGTAGA CAAGGTACAACCC-3' reverse for mouse Tnfa (148 bp); and 5'-GGTAAAGTGGATATTGTTGCCATCAATG-3' forward, and 5'-GGAGGGATCTCGCTCCTGGAAGATGGTG-3' reverse for mouse $G A P D H(173 \mathrm{bp})$. The relative fold expression and changes were calculated $2^{-\Delta \Delta \mathrm{Cq}}$ method (17).

Immunoblotting. Cell culture supernatant from non-treated BMMCs, BMMCs stimulated with LPS $(1 \mu \mathrm{g} / \mathrm{ml})$, and BMMCs stimulated with LPS in the presence of the benzoxazole derivatives (ICP-1, 2, 5, and/or 13) were collected and prepared in a sample buffer [0.5 M Tris/HCl (pH 6.8), 25\% glycerol, $10 \%$ SDS, 500 mM DTT, 1\% bromophenol blue; Sigma-Aldrich; Merck KGaA].

Equal amounts $(40 \mu \mathrm{l})$ cell lysate were resolved by $10 \%$ SDS-PAGE and transferred to Immobilon-P polyvinylidene 
A

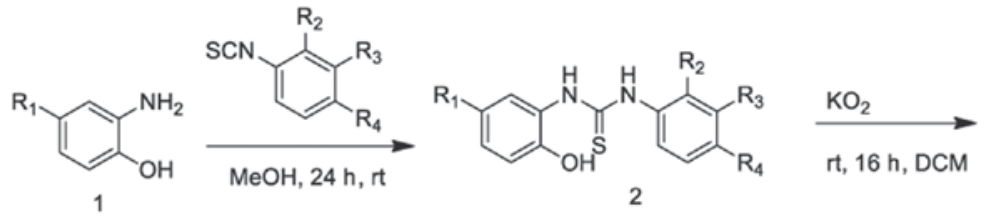

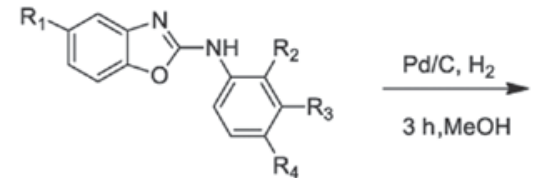

ICP-2 R1= CH3, R2=H, R3=H, R4= $\mathrm{CH} 2 \mathrm{CH} 3$ ICP-13 R1= CH3, R2= OCH3, R3=H, R4=NO2

B

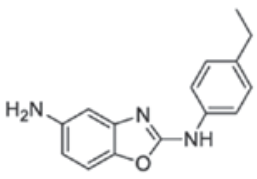

ICP-1

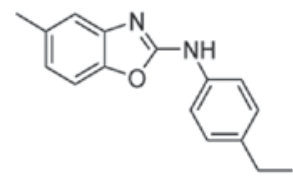

ICP-2

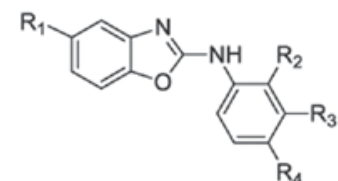

ICP-1 R1 $=\mathrm{NH} 2, \mathrm{R} 2=\mathrm{H}, \mathrm{R} 3=\mathrm{H}, \mathrm{R} 4=\mathrm{CH} 2 \mathrm{CH} 3$

ICP-5 R1 $=$ NH2, R2= H, R3= Br, R4=H

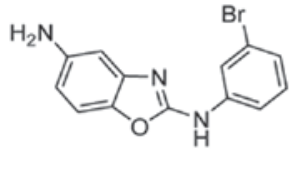

ICP-5

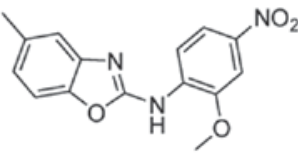

ICP-13

Figure 1. Synthesis of benzoxazole derivatives. (A) Synthetic procedure for benzoxazole derivatives. (B) Structures of ICP-1, ICP-2, ICP-5, and ICP-13. ICP, anti-Itch agent by Park; DCM, dichloromethane; rt, room temperature; Pd/C, palladium on carbon catalyst.

fluoride membranes (EMD Millipore, Billerica, MA, USA). Membranes were blocked with 5\% skim milk in TBS containing $0.1 \%$ Tween-20 (TBS-T) solution for $1 \mathrm{~h}$ at room temperature and were subsequently incubated with primary antibodies overnight at $4{ }^{\circ} \mathrm{C}$. The following primary antibodies were used: IL-1 $\beta$ (1:200, diluted in $3 \%$ bovine serum albumin (BSA; Bovogen Biologicals, Pty, Ltd., East Keilor, Victoria, Australia) containing TBST; cat. no. sc-7884; Santa Cruz Biotechnology, Inc., Dallas, TX, USA); and $\beta$-actin [1:3,000, diluted in 3\% BSA containing TBST; cat. no. A1978; mouse; Sigma-Aldrich; Merck KGaA]. The membranes were washed 3 times for $10 \mathrm{~min}$ in TBST and incubated with anti-rabbit (cat.no. BR170-6515; Bio-Rad Laboratories, Inc.) or anti-mouse (cat. no. BR170-6516; Bio-Rad Laboratories, Inc.) horseradish peroxidase-conjugated secondary antibodies (1:3,000, diluted in TBST) for $1 \mathrm{~h}$ at room temperature. Following incubation, membranes were washed 3 times for $10 \mathrm{~min}$ in TBST and developed using SuperSignal West Femto Maximum Sensitivity Substrate (Pierce; Thermo Fisher Scientific, Inc.). Images were obtained using ImageQuant LAS 4000 (GE Healthcare Life Sciences, Little Chalfont, UK). The band pixel densities of the precursor form of IL- $1 \beta$ and the active form of IL-1 $\beta$ were divided by the pixel densities of the corresponding $\beta$-actin bands for quantitation using UN-SCAN-IT-gel 6.1 software (Silk Scientific Inc., Orem, UT, USA).

ELISA. For histamine measurements, $80 \mathrm{nM}$ phorbol-12-myristate-13-acetate (PMA) (Ebioscience; Thermo Fisher Scientific, Inc.) and ionomycin (1 $\mu \mathrm{M}$; Ebioscience; Thermo Fisher Scientific, Inc.) were added in the presence of the benzoxazole derivatives, and the cultures were maintained for $24 \mathrm{~h}$ at $37^{\circ} \mathrm{C}$ in a humidified atmosphere of $5 \% \mathrm{CO}_{2}$. Cell culture supernatants were collected from non-treated BMMCs and BMMCs stimulated with LPS $(1 \mu \mathrm{g} / \mathrm{ml})$ in the presence of the benzoxazole derivatives (ICP-1, 2, 5, and/or 13). The levels of secreted histamine were determined using a histamine ELISA kit in accordance with the manufacturer's recommended protocol (Abnova; cat. no. KA2589; Taipei, Taiwan).

Statistical analysis. Data are presented as the mean \pm standard error of the mean $(n=3)$. Statistical significance was determined by one-way analysis of variance (ANOVA) in conjunction with Dunnett's post hoc test, or two-way ANOVA in conjunction with Sidak's post-hoc test compared with the normal group using GraphPad Prism, version 7 software (GraphPad Software, Inc., La Jolla, CA, USA). $\mathrm{P}<0.05$ was considered to indicate a statistically significant difference.

\section{Results}

Generation of BMMCs from mice. BMMCs were generated from C57BL/6 mice. As exhibited in Fig. 2A, low expression of the mast cell markers FceRI and c-kit was observed prior to BMMC differentiation on the cell surface of fresh mouse bone marrow cells. However, following 5 weeks of culture in the presence of IL-3, $>90 \%$ of the cells expressed FceRI and c-kit (Fig. 2B). In addition, toluidine blue staining demonstrated differentiated MCs that contained granules in the cytoplasm (Fig. 2C).

Benzoxazole derivatives attenuate the LPS-mediated gene expression of Illb Il6, Il13, Tnfa, Plin2 and Plin3 in BMMCs. A number of studies have indicated that MCs produce proinflammatory cytokines, including IL-1 $\beta$, IL-6, IL-13 and TNF- $\alpha$ when exposed to LPS $(4,18-20)$. LOX activation positively regulates inflammatory responses, including LPS-induced inflammation $(21,22)$ and LPS is a direct inducer of 5-LOX in immune cells, including monocytes (23). As it was reported that benzoxazoles are 5-LOX and IL-6 inhibitors (13), benzoxazole derivatives were synthesized and LPS-stimulated 
A BMCs
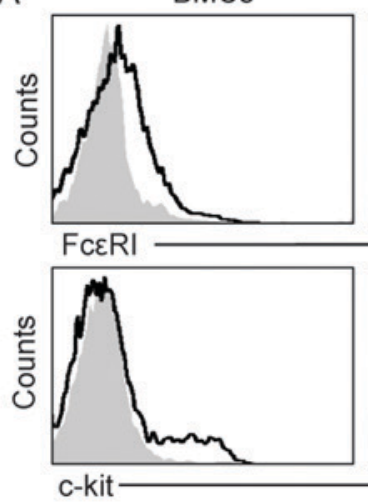

Isotype antibody - Marker
BMMCs
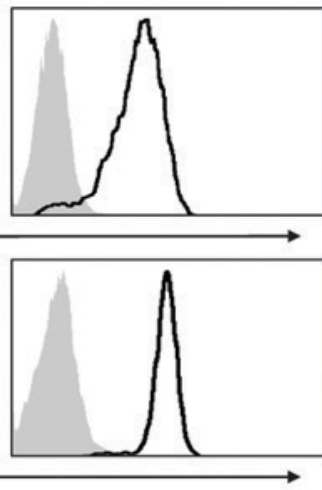

B

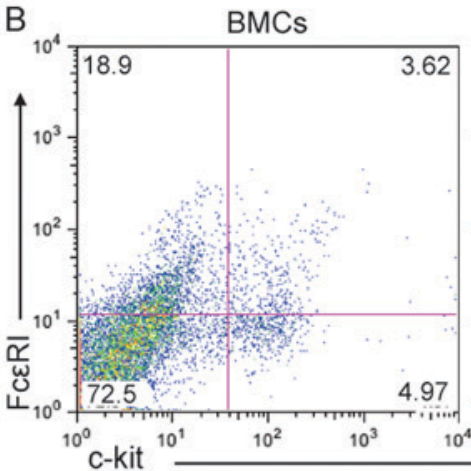

BMMCs

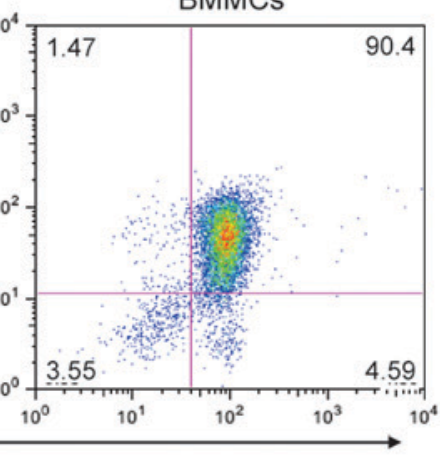

C

BMCs
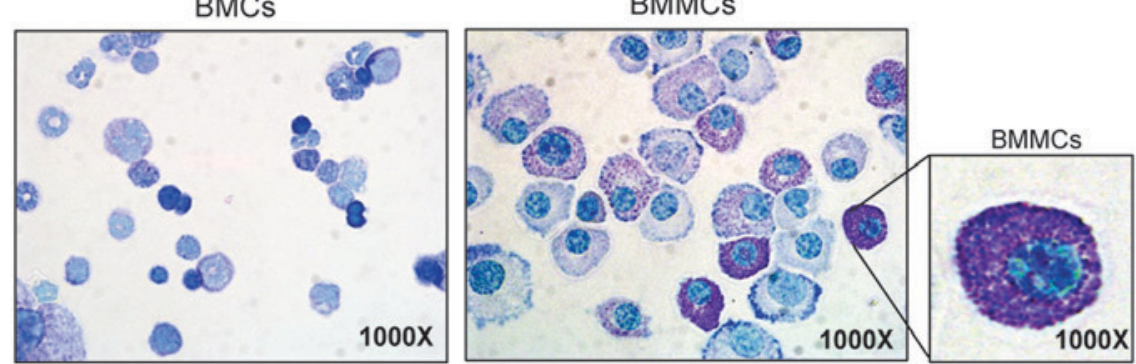

Figure 2. Generation of BMMCs. (A) The surface expression of FceRI and c-kit on BMCs and BMMCs are exhibited as representative histograms using flow cytometry. (B) The surface expression of FceRI and c-kit on BM cells and BMMCs are exhibited as representative dot plots from the flow cytometry. (C) BM cells (left) and BMMC (right) were distinguished by toluidine blue stain. BMMCs, bone marrow-derived mast cells; c-kit, cluster of differentiation 17; BMCs, bone marrow cells; FceRI, high affinity immunoglobulin $\varepsilon$ receptor subunit $\gamma$.

BMMCs were treated with the inhibitors for $24 \mathrm{~h}$. Benzoxazole derivatives had an inhibitory effect on the expression of the Illb, Il6, Il13 and Tnfa genes in BMMCs. In particular, a higher fold increase in $I l 1 b$ and Tnfa expression, compared with Il6 and Il13, following LPS stimulation was observed. A total of three types of cytokines (Illb, Ill3 and Tnfa) were significantly downregulated by all four types of benzoxazole derivatives (ICP-1, 2, 5 and 13; P<0.05; Fig. 3A).

In addition, lipid droplets (LD) from MCs are involved in cell signaling and the generation of biologically active lipid mediators (e.g. prostaglandin D2, leukotriene B4 and C4) evoked by inflammatory and infectious conditions $(24,25)$. The most well-known LD proteins are members of the PLIN family. Out of the five PLINs (PLIN1, PLIN2, PLIN3, PLIN4 and PLIN5), PLIN2 and PLIN3 are expressed in developing and mature human MCs (26), and the constitutive expression of the Plin2 and Plin3 genes in BMMCs was confirmed in the present study (data not shown). A modest, although significant, inhibition and induction of Plin2 and Plin3, respectively by ICP-1 and 5, following treatment with LPS was observed ( $\mathrm{P}<0.05$; Fig. 3A).

As the activation of MCs via the NACHT, LRR and PYD domains-containing protein 3 (NLRP3) inflammasome may be linked to IL-1 $\beta$ production (27), the protein expression levels of IL-1 $\beta$ in the cell culture supernatant were tested. Following $24 \mathrm{~h}$ of treatment with LPS in the presence or absence of benzoxazole derivatives, the secreted active form of IL-1 $\beta$ was most abundant in the BMMC supernatant; however, benzoxazole derivatives suppressed the secretion of the protein. Instead, a precursor form of IL-1 $\beta$ (pro-IL-1 $\beta$ ) accumulated, particularly under treatment with the benzoxazole derivatives ICP-2 and ICP-5 (Fig. 3B).

Benzoxazole derivatives suppress histamine production in $B M M C s$. LPS-induced inflammation was demonstrated to increase histamine secretion, possibly by directly stimulating histamine-forming enzyme (histidine decarboxylase) activity (28). Therefore, it was hypothesized that MC activation by LPS may be followed by histamine secretion. Furthermore, the suppressive effect of the benzoxazole derivatives was additionally investigated. The secretion of histamine from BMMCs was triggered by PMA and ionomycin as a positive control. As exhibited in Fig. 4A, LPS stimulation alone for $24 \mathrm{~h}$ promoted histamine production in BMMCs. Although the increases in histamine production with ICP-1, 2 and 5 were not statistically significant, benzoxazole derivative ICP-13 significantly inhibited the increase $(\mathrm{P}<0.05)$. The BMMCs treated with PMA and ionomycin generated significantly increased histamine levels, which was suppressed by all the inhibitors $(\mathrm{P}<0.05$; Fig. 4B).

Benzoxazole derivatives do not affect the surface expression of co-stimulatory molecules on BMMCs. In addition to their contribution to host defense via innate mechanisms, MCs additionally promote adaptive immune responses through interactions with $\mathrm{CD}^{+}$and $\mathrm{CD}^{+} \mathrm{T}$ cells (29). It was demonstrated that MCs may be primed to express functional class II major histocompatibility complex molecules and co-stimulatory molecules, and may serve as antigen presenting cells for $\mathrm{CD}^{+} \mathrm{T}$ lymphocytes, including helper $\mathrm{T}$ cells (30). Therefore mouse BMMCs were examined by flow cytometry for the 

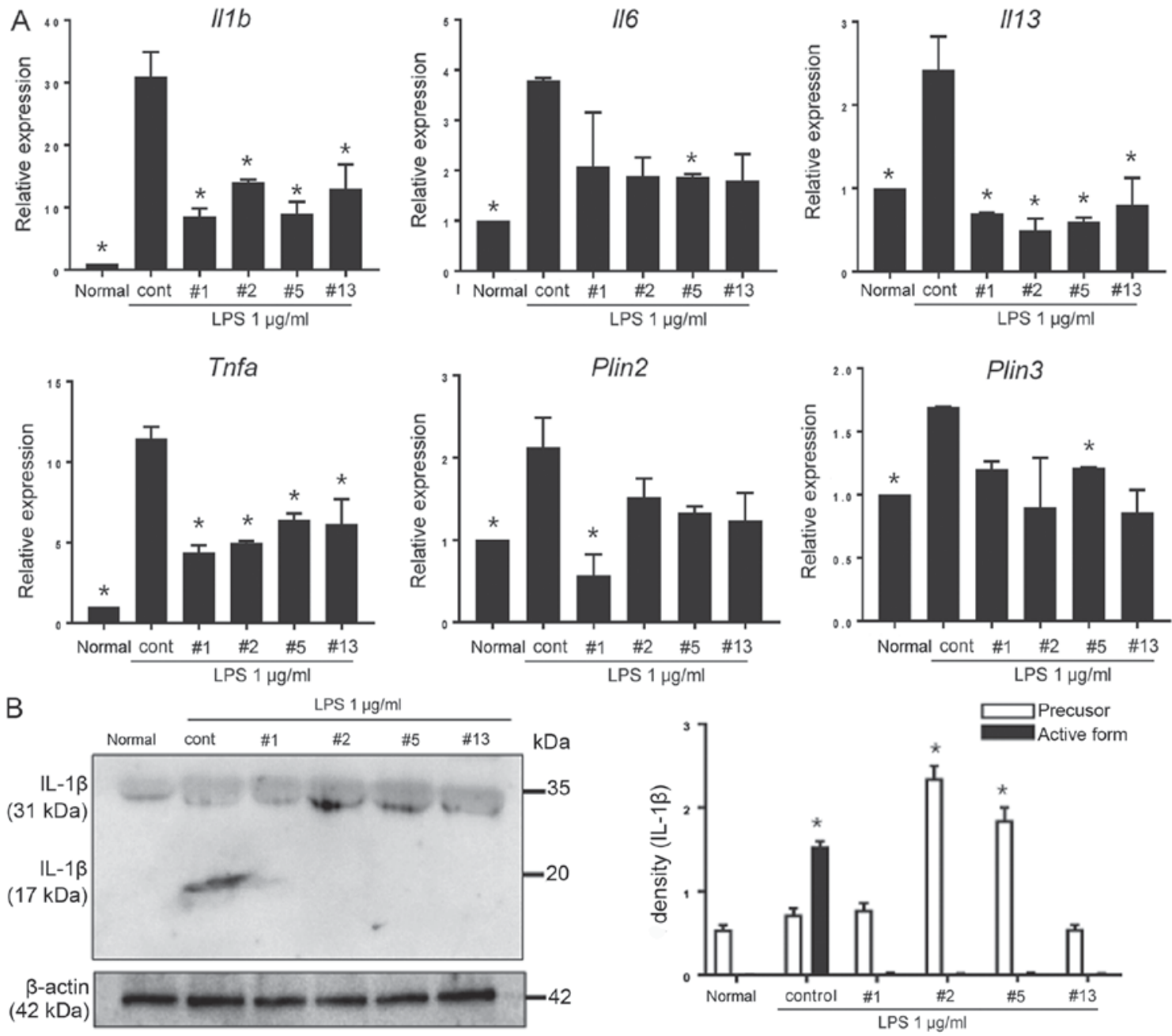

Figure 3. Gene expression profiles in BMMCs. (A) Non-treated normal BMMCs, BMMCs cultured with LPS, and BMMCs cultured with LPS in the presence of benzoxazole derivatives (10 $\mu \mathrm{M}$ each) were collected, and the mRNA expression of Illb, Il6, Ill3, Tnfa, Plin2 and Plin 3 was analyzed by reverse transcription-quantitative polymerase chain reaction. The benzoxazole derivatives ICP-1, ICP-2, ICP-5 and ICP-13 are indicated as \#1, \#2, \#5 and \#13, respectively. Data are presented as the mean \pm SEM. ${ }^{*} \mathrm{P}<0.05$ vs. control. (B) The level of secreted IL- $1 \beta$ from non-treated normal BMMCs, and BMMCs cultured with LPS stimulation and each benzoxazole derivative (10 $\mu \mathrm{M}$ each) were compared using the cell culture supernatant. The precursor form of IL-1 $\beta$ was indicated by the $31 \mathrm{kDa}$ band and the active form of IL-1 $\beta$ was indicated by the $17 \mathrm{kDa}$ band (upper). The pixel densities of each band were divided by the corresponding $\beta$-actin bands for normalization (lower). Data are presented as the mean \pm SEM. ${ }^{*} \mathrm{P}<0.05$ vs. normal. SEM, standard error of the mean; BMMCs, bone marrow derived mast cells; LPS, lipopolysaccharide; Il/IL, interleukin; Tnfa, tumor necrosis factor- $\alpha$; cont, control; Plin, perilipin.
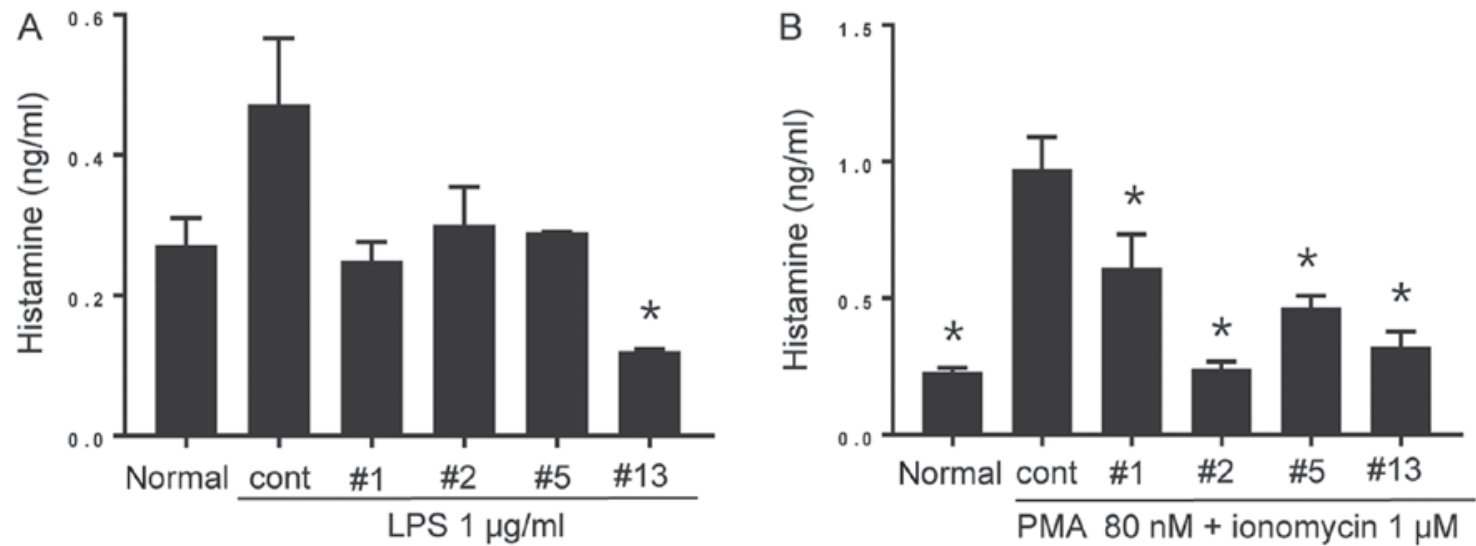

Figure 4. Histamine production of BMMCs. (A) The cell culture supernatant from non-treated normal BMMCs, BMMCs treated with LPS, and BMMCs treated with LPS in the presence of each of the benzoxazole derivatives $(10 \mu \mathrm{M}$ each) for $24 \mathrm{~h}$ was collected and the amount of secreted histamine was measured by ELISA. The benzoxazole derivatives ICP-1, ICP-2, ICP-5 and ICP-13 are indicated as \#1, \#2, \#5 and \#13, respectively. Data are presented as the mean \pm SEM. ${ }^{*} \mathrm{P}<0.05$ vs. cont. (B) Cell culture supernatants from non-treated normal BMMCs, BMMCs treated with PMA and ionomycin, BMMCs treated with PMA and ionomycin in the presence of each benzoxazole derivative $(10 \mu \mathrm{M}$ each) for $24 \mathrm{~h}$ were collected, and the amount of secreted histamine was measured by ELISA. Data are presented as the mean \pm SEM. " $\mathrm{P}<0.05$ vs. cont. SEM, standard error of the mean; BMMCs, bone marrow derived mast cells; LPS, lipopolysaccharide; PMA, phorbol-12-myristate-13-acetate; cont, control; ICP, anti-Itch agent by Park. 

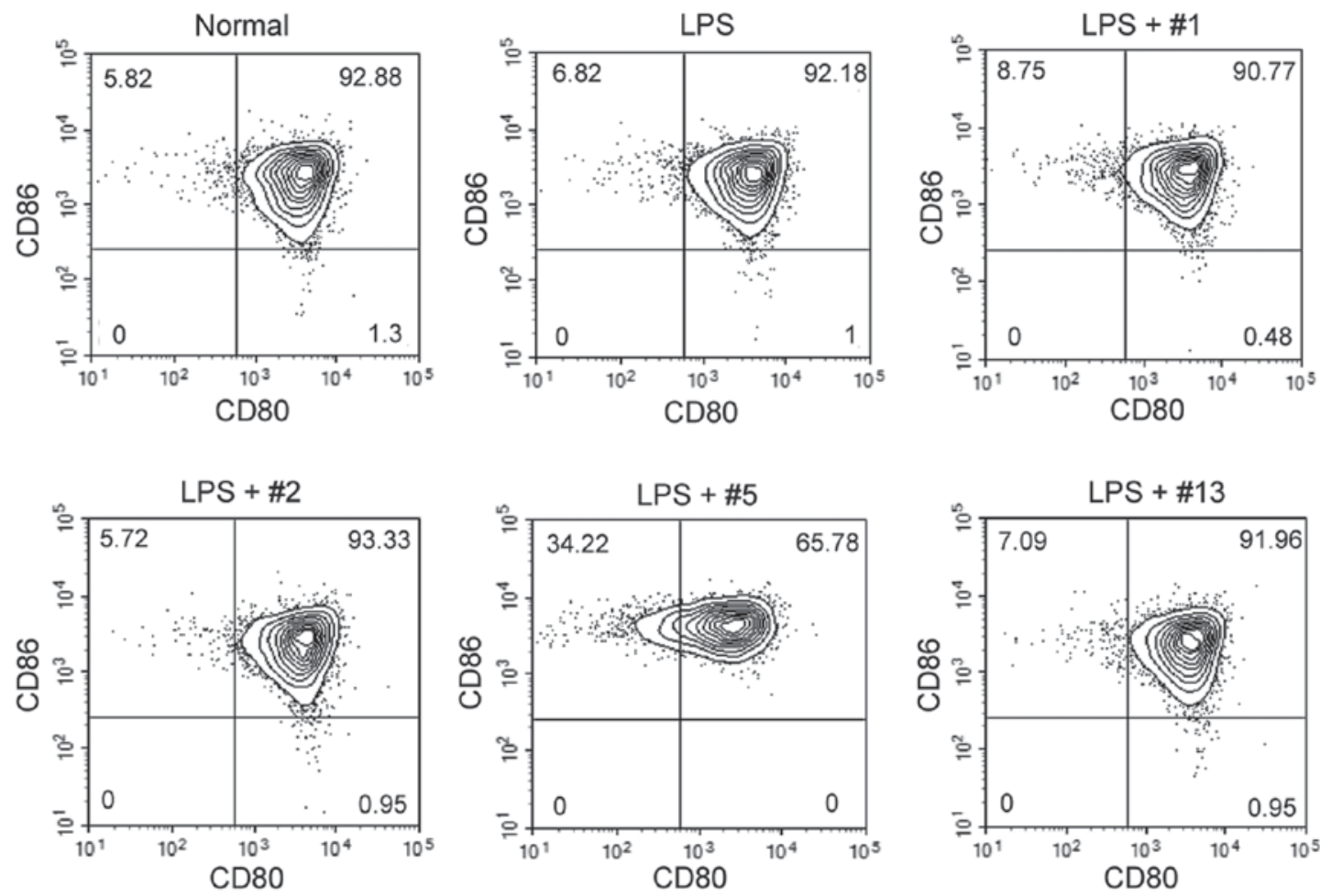

Figure 5. Surface expression of co-stimulatory molecules in BMMCs. Non-treated normal BMMCs, and BMMCs cultured in the presence of LPS $(1 \mu \mathrm{g} / \mathrm{ml})$ and each benzoxazole derivative (10 $\mu \mathrm{M}$ each) for $24 \mathrm{~h}$ were collected, and the surface expression of CD80 and CD86 was analyzed by flow cytometry. The benzoxazole derivatives ICP-1, ICP-2, ICP-5 and ICP-13 are indicated as \#1, \#2, \#5 and \#13, respectively. BMMCs, bone marrow derived mast cells; LPS, lipopolysaccharide; CD, cluster of differentiation, ICP, anti-Itch agent by Park.

surface expression of members of the B7 protein family, CD80 and CD86, which are potent co-stimulatory proteins for $\mathrm{T}$ cell activation. BMMCs highly expressed both CD80 and CD86 even in the absence of stimulation, and their expression was not altered following $24 \mathrm{~h}$ of treatment with LPS. Similarly, simultaneous treatment with benzoxazole derivatives and LPS did not affect the expression level of these surface proteins. However, ICP-5 downregulated the percentages of CD80-positive BMMCs (Fig. 5), by modifying CD80 expression rather than CD86 expression.

\section{Discussion}

Previously, the authors reported that benzoxazoles act as 5-LOX or IL-6 inhibitors (13-16). As part of the authors' ongoing study on the use of benzoxazoles as anti-inflammatory agents, benzoxazole derivatives were synthesized and their effects on MCs were determined. In the present study, benzoxazole derivatives were demonstrated to attenuate LPS-induced BMMC activation. Specifically, it was demonstrated that the LPS-mediated gene expression of $I l 1 b, I l 6$, Ill3, Tnfa, Plin2 and Plin 3 in BMMCs was downregulated by four benzoxazole derivatives. Furthermore, histamine secretion from BMMCs exposed to LPS or PMA/ionomycin was suppressed in the presence of benzoxazole derivatives. However, the inhibition of the surface expression of co-stimulatory molecules, including CD80 and CD86, was less evident.

MCs are particularly abundant in the skin, airway and gut mucosa, where they are strategically located for optimal interaction with the environment. MCs are heterogeneous in terms of morphology, receptor expression, mediator content and reactivity towards various stimulants, according to their localization in the body and among animal species. Therefore, cellular pathways acting on MCs to produce key mediators involved in inflammation, regardless of the heterogeneity of cells, may be efficient targets to modulate MC responses (31). MCs have been demonstrated to express the majority of TLRs, and to respond to their agonists by secreting cytokines, chemokines and lipid mediators. There are similarities and differences between the cytokines produced upon stimulation with different TLRs. For example, TLR2 and TLR4 trigger the production of the Th2 cytokines IL-4, IL-5, IL-13, IL-6 and TNF- $\alpha$ from MCs via the c-Jun N-terminal kinase and p38 pathways (4). Additionally, the benzoxazole derivatives, particularly ICP-1, used in the present study were reported to suppress the production of interferon- $\gamma$, IL-17, IL-4, IL-5 or IL-13 via suppression of the IL-6-signal transducer and activator of transcription 3 signaling pathway (16).

LPS stimulation of BMMCs induced the expression of $I l l b$ mRNA in addition to the induction of the active form of IL-1 $\beta$, which was suppressed by the benzoxazole derivatives. Notably, treatment with benzoxazole derivatives failed to induce expression of the cleaved form of active IL-1 $\beta$. This result suggested that the benzoxazole derivatives used in the present study may inhibit the activation of the NLRP3 signaling pathway directly or indirectly, via a decrease in TNF- $\alpha$ (32) or PLIN (33) from BMMCs. Activation of the NLRP3 inflammasome requires two signals: Signal 1 from microbial components (e.g. LPS) or endogenous cytokines (e.g. TNF- $\alpha$ ), and signal 2 from 
adenosine triphosphate or bacterial toxin stimulation of the NLRP3 inflammasome. Signal 1 leads to the upregulation of NLRP3 in addition to pro-IL-1 $\beta$ expression via nuclear factor $(\mathrm{NF})-\kappa \mathrm{B}$ activation through TLR4. Signal 2 leads to the activation of the NLRP3 inflammasome followed by activation of caspase 1 , which cleaves pro-IL-1 $\beta$ to the active form of IL-1 $\beta$. Therefore, the benzoxazole derivatives that were tested possibly inhibited the $\mathrm{NF}-\kappa \mathrm{B}$ pathway and/or inflammasome activation, either by a direct effect on the $\mathrm{NF}-\kappa \mathrm{B}$ signaling pathway or an indirect effect by suppressing 5-LOX activity (13).

Inflammation is a fundamental protective response in higher eukaryotes against a variety of external stimuli, including environmental toxins, pathogens and allergens. These stimuli are encountered by immune cells including neutrophils, MCs or macrophages, which mediate the initial defense reaction. Therefore, the response of these cells upon activation is indispensable for maintaining host defenses and homeostasis. However, excessive or unresolved activation may induce pathophysiological processes resulting in the development of inflammatory disease. Inflammation is closely associated with oxidative processes and, therefore, oxidative enzymes, including 5-LOX, that are known to serve key roles in inflammation (34). 5-LOX generates a number of lipid and pro-inflammatory mediators that directly act on inflammation. Furthermore, the mediators generated by 5 -LOX including LTs are involved in the activation of pro-inflammatory signal transduction pathways including the $N F-\kappa B$ signaling pathway that potentiates inflammatory status. Indeed, 5-LOX-generated pro-inflammatory products have been implicated in a number of human acute and chronic inflammatory diseases, including asthma, atherosclerosis, rheumatoid arthritis, inflammatory bowel diseases, urticaria and atopic dermatitis.

It was reported that 5-LOX enzymatic activity is inhibited by phenolic antioxidants including nordihydroguaiaretic acid and caffeic acid, suggesting a beneficial role of dietary polyphenol intake (35). Synthetic drugs that act on LOX are currently relatively limited. The 5-LOX inhibitor zileuton has been used successfully for the control of asthma. Currently, 5-LOX inhibitors are being developed by pharmaceutical companies (36). These inhibitors prevent the transportation of 5-LOX from the nucleus to the cytoplasm, leading to the suppression of 5-hydroperoxyeicosatetraenoic acid production.

In conclusion, the present study demonstrated that benzoxazole derivatives (ICP-1, 2, 5 and 13) suppressed the production of proinflammatory cytokines of BMMCs and LD proteins, including PLINs, following LPS stimulation. Therefore, agents, including benzoxazole derivatives that are able to inhibit acute inflammation resulting from the activation of MCs in allergic reactions or urticaria may be therapeutically important. Further studies are required to refine the mode of action of benzoxazole derivatives and their therapeutic effects in vivo to determine the most effective therapeutic strategy.

\section{Acknowledgements}

Not applicable.

\section{Funding}

The present study was supported by the Institute of Clinical Medicine Research of Bucheon St. Mary's Hospital, Research Fund, BCMC12AH08. In addition, the present study was supported by the RP-Grant 2018 of Ewha Womans University.

\section{Availability of data and materials}

The datasets used and/or analyzed during the current study are available from the corresponding author on reasonable request.

\section{Authors' contributions}

KAC performed experiments and wrote the manuscripts. MP and YHK performed experiments and analyzed data. HP and KHL designed the experiments and wrote the manuscripts.

\section{Ethics approval and consent to participate}

All procedures were approved by the Ewha Womans University College of Medicine Animal Care and Use Committee (Seoul, Korea; ESM 15-0309).

\section{Consent for publication}

Not applicable.

\section{Competing interests}

The authors declare they have no competing interests.

\section{References}

1. Sismanopoulos N, Delivanis DA, Alysandratos KD, Angelidou A, Therianou A, Kalogeromitros D and Theoharides TC: Mast cells in allergic and inflammatory diseases. Curr Pharm Des 18: 2261-2277, 2012.

2. Wernersson S and Pejler G: Mast cell secretory granules: Armed for battle. Nat Rev Immunol 14: 478-494, 2014

3. Yamashita M and Nakayama T: Progress in allergy signal research on mast cells: Regulation of allergic airway inflammation through toll-like receptor 4-mediated modification of mast cell function. J Pharmacol Sci 106: 332-335, 2008

4. Masuda A, Yoshikai Y, Aiba K and Matsuguchi T: Th2 cytokine production from mast cells is directly induced by lipopolysaccharide and distinctly regulated by c-Jun N-terminal kinase and p38 pathways. J Immunol 169: 3801-3810, 2002.

5. Murakami D, Yamada H, Yajima T, Masuda A, Komune S and Yoshikai Y: Lipopolysaccharide inhalation exacerbates allergic airway inflammation by activating mast cells and promoting Th2 responses. Clin Exp Allergy 37: 339-347, 2007.

6. Nigo YI, Yamashita M, Hirahara K, Shinnakasu R, Inami M, Kimura M, Hasegawa A, Kohno Y and Nakayama T: Regulation of allergic airway inflammation through Toll-like receptor 4-mediated modification of mast cell function. Proc Natl Acad Sci USA 103: 2286-2291, 2006.

7. Shiba E, Izawa K, Kaitani A, Isobe M, Maehara A, Uchida K, Maeda K, Nakano N, Ogawa H, Okumura K, et al: Ceramide-CD300f binding inhibits lipopolysaccharide-induced skin inflammation. J Biol Chem 292: 2924-2932, 2017.

8. Kuhn $\mathrm{H}$ and O'Donnell VB: Inflammation and immune regulation by 12/15-lipoxygenases. Prog Lipid Res 45: 334-356, 2006

9. Han H, Liang X, Ekberg M, Kritikou JS, Brunnström A, Pelcman B, Matl M, Miao X, Andersson M, Yuan X, et al: Human 15-lipoxygenase-1 is a regulator of dendritic-cell spreading and podosome formation. FASEB J 31: 491-504, 2017. 
10. Claesson HE: On the biosynthesis and biological role of eoxins and 15-lipoxygenase-1 in airway inflammation and Hodgkin lymphoma. Prostaglandins Other Lipid Mediat 89: 120-125, 2009.

11. Ro M, Lee AJ and Kim JH: 5-/12-lipoxygenase-linked cascade contributes to the IL-33-induced synthesis of IL-13 in mast cells, thus promoting asthma development. Allergy 73: 350-360, 2018.

12. Mashima R and Okuyama T: The role of lipoxygenases in pathophysiology; new insights and future perspectives. Redox Biol 6 : 297-310, 2015 .

13. Song H, Oh SR, Lee HK, Han G, Kim JH, Chang HW, Doh KE, Rhee HK and Choo HY: Synthesis and evaluation of benzoxazole derivatives as 5-lipoxygenase inhibitors. Bioorg Med Chem 18 $7580-7585,2010$

14. Lee JH, An MH, Choi EH, Choo HYP and Han G: A facile synthesis of 2-acyl and 2-alkylaminobenzimidazoles for 5-lipoxygenase inhibitors. Heterocycles 70: 571-580, 2006.

15. Yoon JH, Song H, Kim SW, Han G and Choo HYP: A facile synthesis of 2-aminothiazolo [5,4-b]pyridines and 2-aminobenzoxazoles via cyclization of thioureas. Heterocycles 65 2729-2740, 2005.

16. Kim D, Won HY, Hwang ES, Kim YK and Choo HP: Synthesis of benzoxazole derivatives as interleukin-6 antagonists. Bioorg Med Chem 25: 3127-3134, 2017.

17. Livak KJ and Schmittgen TD: Analysis of relative gene expression data using real-time quantitative PCR and the 2(-Delta Delta C(T)) method. Methods 25: 402-408, 2001.

18. Chiba N, Masuda A, Yoshikai Y and Matsuguchi T: Ceramide inhibits LPS-induced production of IL-5, IL-10, and IL-13 from mast cells. J Cell Physiol 213: 126-136, 2007.

19. Hochdörfer T, Tiedje C, Stumpo DJ, Blackshear PJ, Gaestel M and Huber M: LPS-induced production of TNF- $\alpha$ and IL-6 in mast cells is dependent on p38 but independent of TTP. Cell Signal 25: 1339-1347, 2013.

20. Sandig H and Bulfone-Paus S: TLR signaling in mast cells: Common and unique features. Front Immunol 3: 185, 2012.

21. Lopes DEM, Jabr CL, Dejani NN, Saraiva AC, de Aquino SG Medeiros AI and Junior CR: Inhibition of 5-lipoxygenase (5-Lo) attenuates inflammation and bone resorption in lipopolysaccharide (Lps)-induced periodontal disease. J Periodontol: 1-18, 2017 (Epub ahead of print).

22. Rossaint J, Nadler JL, Ley $\mathrm{K}$ and Zarbock A: Eliminating or blocking 12/15-lipoxygenase reduces neutrophil recruitment in mouse models of acute lung injury. Crit Care 16: R166, 2012.
23. Lee SJ, Seo KW and Kim CD: LPS increases 5-LO expression on monocytes via an activation of Akt-Sp1/NF- $\alpha \mathrm{B}$ pathways. Korean J Physiol Pharmacol 19: 263-268, 2015.

24. D'Avila H, Maya-Monteiro CM and Bozza PT: Lipid bodies in innate immune response to bacterial and parasite infections. Int Immunopharmacol 8: 1308-1315, 2008.

25. Bozza PT, Bakker-Abreu I, Navarro-Xavier RA and Bandeira-Melo C: Lipid body function in eicosanoid synthesis: An update. Prostaglandins Leukot Essent Fatty Acids 85: 205-213, 2011.

26. Dichlberger A, Schlager S, Lappalainen J, Käkelä R, Hattula K, Butcher SJ, Schneider WJ and Kovanen PT: Lipid body formation during maturation of human mast cells. J Lipid Res 52: 2198-2208, 2011

27. Nakamura Y, Franchi L, Kambe N, Meng G, Strober W and Núñez G: Critical role for mast cells in interleukin-1 $\beta$-driven skin inflammation associated with an activating mutation in the nlrp3 protein. Immunity 37: 85-95, 2012.

28. Shoji N, Yoshida A, Yu Z, Endo Y and Sasano T: Lipopolysaccharide stimulates histamine-forming enzyme (histidine decarboxylase) activity in murine dental pulp and gingiva. Arch Oral Biol 51: 856-860, 2006.

29. Hershko AY and Rivera J: Mast cell and T cell communication; amplification and control of adaptive immunity. Immunol Lett 128: 98-104, 2010.

30. Kambayashi T, Allenspach EJ, Chang JT, Zou T, Shoag JE, Reiner SL, Caton AJ and Koretzky GA: Inducible MHC class II expression by mast cells supports effector and regulatory $\mathrm{T}$ cell activation. J Immunol 182: 4686-4695, 2009.

31. Espinosa E and Valitutti S: New roles and controls of mast cells. Curr Opin Immunol 50: 39-47, 2017.

32. Nakamura $\mathrm{Y}$ and Kambe N: Linkage of bacterial colonization of skin and the urticaria-like rash of NLRP3-mediated autoinflammatory syndromes through mast cell-derived TNF- $\alpha$. J Dermatol Sci 71: 83-88, 2013.

33. Cho KA and Kang PB: PLIN2 inhibits insulin-induced glucose uptake in myoblasts through the activation of the NLRP3 inflammasome. Int J Mol Med 36: 839-844, 2015.

34. Wisastra R and Dekker FJ: Inflammation, cancer and oxidative lipoxygenase activity are intimately linked. Cancers (Basel) 6: 1500-1521, 2014.

35. Werz O: Inhibition of 5-lipoxygenase product synthesis by natural compounds of plant origin. Planta Med 73: 1331-1357, 2007.

36. Pettersen D, Davidsson Ö and Whatling C: Recent advances for FLAP inhibitors. Bioorg Med Chem Lett 25: 2607-2612, 2015. 